\title{
An approach to the optimal timber transport scheduling
}

\author{
Anton Shabaev ${ }^{1}$, Anton Sokolov ${ }^{1,}{ }^{*}$, Alexander Urban $^{1}$, and Dmitry Pyatin ${ }^{1}$ \\ ${ }^{1}$ Petrozavodsk State University, 185910 Lenin av., 33, Petrozavodsk, Russian Federation
}

\begin{abstract}
An approach to the optimal timber transport scheduling is described in the paper. A description of this problem is given, a multi-criteria mathematical model is created. It is noted that the problem belongs to the class of General vehicle routing problems (GVRP) associated with the jobshop scheduling. A hybrid algorithm for solving this problem based on the decomposition method using the simplex method and the genetic algorithm is developed. Testing of the proposed approach using real data from wood harvesting enterprises showed its effectiveness. The algorithm was implemented in "Opti-Wood" decision support system for wood harvesting planning and management, developed by Opti-Soft company (Russia).
\end{abstract}

\section{Introduction}

The importance of new logistics solutions in the forest industry has grown significantly in Russia in recent years. The optimal scheduling of timber transport is a key problem in the operational management of wood harvesting company. In the case of wood harvesting company, this problem is especially difficult compared to other industries. This is primarily due to the accelerated growth of harvesting volumes carried out using cut-to-length technology. The use of the cut-to-length technology significantly complicates finding the optimal transport plan, since its classical scheme does not include large centralized forest yards, and there is a significant increase in the range of wood assortments produced at each harvesting site. The roundwood is transported directly to customers, the number of which can be quite large $[1,2]$.

This paper describes the problem of optimal scheduling of timber transportation. A mathematical model of the problem is formulated. The original problem was reduced to two sequentially related subproblems: 1) the optimal distribution of ordered volumes between supply points and machinery sets; 2) the timber transport machines routing. The authors propose a hybrid solution method based on the simplex method, genetic and "greedy" algorithms [3]. The calculation results based on real data from wood harvesting companies are presented.

\footnotetext{
* Corresponding author: a sokolov@petrsu.ru
} 


\section{Problem description}

Scheduling of the timber transport is the timber trucks routing problem with a short planning horizon (1-7 days). It is necessary to schedule the transportation of roundwood for a known set of consumer orders, supply points, demand points, the machinery sets used and production schedule at the supply points. Scheduling is carried out on a given road network in volumes corresponding to consumer's orders, with minimization of undelivered volume, inoperative time and empty run, and taking into account multiple technological constraints (driver breaks, demand points operating hours, capacity of machinery sets, etc.). This task belongs to the class of General Vehicle Routing Problems (GVRP) associated with the job-shop scheduling [4]. The general statement of GVRP requires combining many variants of the original VRP task (VRPTW, VRPPD, etc.) with various constraints (on time windows, on multiple visits to supply points, etc.) [5]. One of the most promising and frequently used approaches to solving this problem is a hybrid method, which is a synthesis of exact and metaheuristic algorithms $[6,7]$.

The main objects used in the problem statement can be described as follows:

1. Demand point is a customer's yard, or a terminal for products storing, characterized by: periods of work; location.

2. Supply point is the pair "harvesting site - assortment", which is determined by: the volume of the assortment stored at the harvesting site or intermediate store on the planning start date; volume increase for each day of the planning period associated with ongoing harvesting operations; location.

3. Order - the consumer's roundwood supply order characterized by such parameters as: price (rubles / cub. m); volume (cub. m); assortment; demand point; timber truck cargo capacity adjustment coefficient - a numerical indicator reflecting the dependence between the transported volume of roundwood and specific tree species and assortment.

4. Machinery set - timber truck, consisting of a vehicle equipped with a platform for roundwood and a trailer, having the following parameters: vehicle capacity (cub. m); trailer capacity (cub. m); list of breaks and shift changes; average loading and unloading time (min); starting location; garage location.

5. Elementary run - a single route of a machinery set from supply points to demand points, at which initially supply points are visited sequentially, and then the machinery set is traveling to the demand point. If there is more than one supply point in the elementary run, the run is called "collecting", otherwise - "complete". The elementary run is characterized by: a machinery set; a sequence of supply points with known loading volumes; demand point.

6. Road network is determined by the graph $G=<V, E>$, where $E$ - the set of road sections, $V$ - the set of points on the map. A road network description method is given in [8].

The problem is to determine the volume for transporting from each supply point to each demand point that maximizes the roundwood sale profits, and generate an operational schedule for timber trucks according to the given road network with the criteria of:

- minimization of the undelivered volume,

- minimization of the inoperative time and empty run.

The following production constraints are taken into account: the schedule of machinery sets (breaks, shift changes, weekends); operation periods of demand points; various road network restrictions; the assortment transported on the vehicle platform may differ from the assortment transported in the trailer within one elementary run; minimum percentage of 
machinery set loading; maximum number of loads in a collecting run; control time determines the duration of the control operation after loading.

\section{Mathematical model}

The index sets of the mathematical model are defined as follows:

$F$ - set of machinery sets, $f \in F$

$I$ - set of supply points, $i \in I$;

$J-$ set of orders, $j \in J$

$R$ - set of demand points (set of terminals), $r \in R$

$J_{r}$ - set of orders associated with the terminal, $r \in R$;

$D$ - set of days in the planning period, $d \in D$

$D_{j} \subset D$ - delivery period on order $j$.

Parameters:

$b_{j}-$ required volume of assortment according to order $j$;

$c_{j}$-assortment price per 1 cub. m. according to order $j$;

$n_{j}^{J}$ - assortment according to order $j$;

$u_{r}$ - total volume of the assortment at terminal $r$ before the start of the planning period;

$w_{r}$ - capacity of the terminal $r$;

$\rho_{r}-$ throughput of the terminal $r$;

$v_{i}$ - total volume at the supply point $i$ before the start of the planning period;

$n_{i}^{I}$ - assortment according to supply point $i$;

$m_{i d}$ - assortment volume producing at the supply point $i$ during the day $d$;

$c_{i f f}$ - transporting costs for $1 \mathrm{cub}$. $\mathrm{m}$ of assortment from supply point $i$ to demand point according to order $j$ for machunery set $f$ during the day $d$ taking into account the periods of road closures and dates of entry into operation of the several road sections.

$p_{f}$ - average capacity of the machinery set $f$;

$k_{j}$ - cargo capacity adjustment coefficient for assortment according to order $j$.

$h_{f d}$ - number of operating hours of the machinery set $f$ during the day $d$ according to its calendar schedule;

$t_{i j f}$ - transportation time from supply point $i$ to demand point $j$ (including times for loading, control and unloading) for the machinery set $f$ during the day $d$;

$Q$ - maximum number of loads within the "collecting" elementary run;

$P$ - minimum percentage of machinery set loading;

$t_{f}^{S}-$ start time for the machinery set $f$;

$p_{f}^{S}-$ curent location of the machinery set $f$.

Unknown factors:

$E$ - set of elementary runs $\left\{\left(T_{e}, L_{e}, f, j_{e}, d_{e}\right)\right\}, e \in E$, where

$T_{e}=\left[t_{e}^{1}, t_{e}^{2}\right]-$ period of the run (runs of one machinery set are ordered by time and do not intersect);

$L_{e}-$ ordered index set of loads within the run $\left\{\left(i_{l}, v_{l}\right)\right\}$, consisting of supply points $i_{l}$ and volumes $v_{i}, l \in L_{e}$;

$f_{e} \in F-$ machinery set performing run $e$; 
$j_{e} \in J-$ order for the run $e$;

$d_{e} \in D-$ start day for the run $e$.

Let all loading within an elementary run be made within one day. Then:

$E_{d} \subset E-$ set of runs during that day $d, d \in D$;

$E_{j} \subset E-$ set of runs for order $j, j \in J$;

$E_{f} \subset E$ - ordered set of runs carried out by machinery set $f, f \in F$;

$L_{e i} \subset L_{e}$ - ordered index set of loads within the run $e$ with supply point $i$;

$V_{e}=\sum_{l \in L_{e}} v_{l}-$ total transporting volume within the run.

Calculated parameters and functions:

$t_{e f}^{w}(\tau)$ - estimated sum of waiting times for accumulating the required volume at supply points, the waiting time before opening of demand points and the downtime in the garage before shift changing for the run $e$;

$t_{e f}(\tau)$ - estimated driving time of the machinery set $f$ for the run $e$ (including loading, control operations, moving, unloading), starting from time $\tau$ taking into account the operating hours of demand points, breaks and shift changes of machinery set, the days and hours of operation of the machinery set, as well as the waiting time for accumulating the required volume $t_{e f}^{w}(\tau)$;

$t_{e f}^{m}(\tau)$ - estimated empty run time of the machinery set $f$ from the previous location to the starting point of loading within the run $e$ starting from time $\tau$, including the sum of the waiting time for accumulating the required volume and the downtime in the garage before shift changing. The previous location of the machinery set is determined either by the initial location of the machinery set $p_{f}^{S}$ (for the first run), or by the demand point of the previous run.

The objective functions are represented by expressions (1) - (2) and are given in order of priority:

$$
\begin{aligned}
& \sum_{e \in E} \sum_{l \in L_{e}}\left(c_{j}-c_{i j f d}\right) v_{l} \rightarrow \max \\
& i=i_{l}, j=j_{e}, d=d_{e}, f=f_{e}
\end{aligned}
$$

- maximization of sales profit minus transportation costs;

$$
\sum_{f \in F}\left(t_{e f}^{m}\left(t_{f}^{S}\right)+\sum_{e \in E_{f}}\left(t_{e f}^{w}\left(t_{e}^{1}\right)+t_{e f}^{m}\left(t_{e}^{2}\right)\right)\right) \rightarrow \min
$$

- minimization of the inoperative time and empty run.

Constraints:

$$
\sum_{e \in E_{j}} V_{e} \leq b_{j}, j \in J
$$

- limit on the required volumes of supplies to consumers; 


$$
\sum_{\tau \leq d} \sum_{e \in E_{\tau}} \sum_{l \in L_{e i}} v_{l} \leq v_{i}+\sum_{\tau \leq d} m_{i \tau}, i \in I, d \in D
$$

- limit on the volume of available assortment at supply points for every day;

$$
\sum_{e \in E_{j}: d_{e} \notin D_{j}} V_{e}=0, j \in J
$$

- limit on the transportation of assortments outside the delivery period of orders;

$$
\sum_{e \in E_{j}} \sum_{l \in L_{e i}} v_{l}=0, n_{i}^{I} \neq n_{j}^{J}, i \in I, j \in J
$$

- limit on the balance of supply and demand points for the assortment;

$$
\sum_{e \in E_{f} \cap E_{d}}\left|T_{e}\right| \leq h_{f d}, f \in F, d \in D
$$

- limit on the capacity of machinery sets;

$$
\sum_{j \in J_{r},} \sum_{e \in E_{j} \cap E_{d}} V_{e} \leq w_{r}-u_{r}+d \rho_{r}, r \in R, d \in D
$$

- limit on the capacity and throughput of the terminal;

$$
\begin{gathered}
t_{a}^{1}=t_{f}^{S}+t_{a f}^{m}\left(t_{f}^{S}\right) \\
t_{c}^{1}=t_{b}^{2}+t_{c f}^{m}\left(t_{b}^{2}\right) \\
t_{c}^{2}=t_{c}^{1}+t_{c f}\left(t_{c}^{1}\right) \\
a=e_{1}, b=e_{k-1}, c=e_{k} \\
k=2 . .\left|E_{f}\right|, e_{k} \in E_{f}, f \in F
\end{gathered}
$$

- limits on the time of runs;

$$
\left|L_{e}\right| \leq Q, e \in E
$$

- limit on the maximum number of loads within the "collecting" run;

$$
\begin{gathered}
V_{e}=k_{j} \cdot p_{f}, f=f_{e}, j=j_{e}, e \in E,\left|L_{e}\right|=1 \\
P \cdot k_{j} \cdot p_{f} \leq V_{e} \leq k_{j} \cdot p_{f}, f=f_{e}, e \in E,\left|L_{e}\right|>1
\end{gathered}
$$

- limits on the volumes of "complete" and "collecting" run;

$$
v_{l} \geq 0, l \in L_{e}, e \in E
$$

- requirement of non-negativity.

\section{Model analysis and solution method}

The problem defined by the mathematical model (1) - (12) is NP-hard, however, heuristic and metaheuristic methods allow to obtain suboptimal solutions within acceptable time and sufficient for practical application. At the same time, a significant improvement in terms of the solution time and accuracy can be achieved using hybrid methods.

The authors propose a hybrid approach based on the decomposition of the original problem into two sequentially related subproblems: 
1) optimal distribution of ordered volumes between supply points and machinery sets with linearization of part of the constraints, and solving the resulting LP problem using exact methods;

2) synthesis of cyclic routes using approximate metaheuristic methods.

\subsection{Optimal distribution of ordered volumes between supply points and machinery sets}

This problem arises by replacing constraint (7) with

$$
\begin{gathered}
\sum_{e \in E_{f} \cap E_{d}} \sum_{l \in L_{e}} \frac{t_{i j f d}}{k_{j} \cdot p_{f}} v_{l} \leq h_{f d} \\
i=i_{l}, j=j_{e}, d=d_{e}, f=f_{e}
\end{gathered}
$$

and highlighting the mathematical model (1), (3) - (6), (8), (10) - (13), which can be written in a linear form by aggregating runs into variables $x_{i j f d}$ - volumes of assortments transported by machinery set $f$ from supply point $i$ to the demand point $j$ during the day $d$.

Described problem is a modification of the well-known transportation problem of linear programming, which can be solved by using simplex method [9-10]. The resulting $x_{i j f d}$ solution must be divided into runs, taking into account technological requirements. Due to the limitation of the minimum machinery set load, exact distribution of volumes is not always achieved, therefore, it is also necessary to minimize the volumes deviations.

To divide the transporting volumes into runs, taking into account constraints (10) - (12), the authors developed a "greedy" algorithm. Runs are formed in the algorithm for fixed day $d$ and machinery set $f$, in the following two stages:

\section{Algorithm 1 Generation of elementary runs}

1. Generation of "complete" runs for each machinery set $f$.

Exactly $n_{i j f d}=\frac{x_{i j f d}}{k_{j} \cdot p_{f}}$ runs of fully loaded machinery set from supply point $i$ with assortment according to order $j$ are created for each pair $i \in I, j \in J$.

2. Generation of "collecting" runs for each machinery set $f$.

After the first stage is completed, for some sets $(i \in I, j \in J, f \in F, d \in D$ ) there may remain volumes that are strictly less than the cargo capacity of the machinery set:

$$
y_{i j f d}=x_{i j f d}-n_{i j f d} \cdot k_{j} \cdot p_{f}<k_{j} \cdot p_{f}
$$

The volumes for supply points are:

$$
q_{i}=\sum_{j \in J} y_{i j f d}, i \in I
$$

Transportation of these volumes must be carried out in "collecting" runs taking into account restrictions (10) - (12). The generation of "collecting" runs consists of the following steps: 


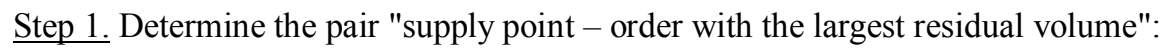

$$
(i, j)=\underset{a \in I, b \in J}{\arg \max }\left\{y_{a b f d}\right\} .
$$

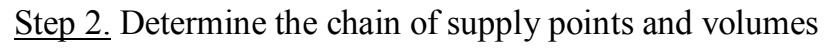

$$
\Omega=\left(\left(i_{1}, v_{1}\right),\left(i_{2}, v_{2}\right), . .,\left(i_{n}, v_{n}\right)\right)
$$

such that:

* $i_{1}=i$

$* n_{i_{1}}^{I}=n_{i_{2}}^{I}=\ldots=n_{i_{n}}^{I}-$ the same assortment at supply points;

* $i_{k}=\underset{a \in I}{\arg \min }\left\{w_{i_{k-1, a}}\right\}$, где $w_{a b}-$ minimum distance between supply points $a$ and $b$,

$a, b \in I, k=2 . . n$;

$* v_{k}=\min \left\{q_{i_{k}}, k_{j} \cdot p_{f}-\sum_{l=1}^{k-1} v_{l}\right\}, k=1 . . n$;

* $n \leq Q-$ constraint (10);

$* \sum_{k=1}^{n} v_{k} \geq P \cdot k_{j} \cdot p_{f}-$ constraint (11).

If $\Omega=\varnothing$, then exit the algorithm.

On the basis of $\Omega$, a new run $e$ is formed.

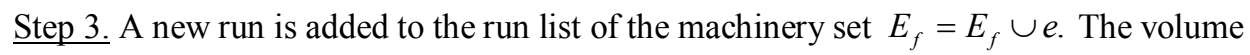
of residues at the supply points $q_{i}$ is reduced in accordance with the volumes of the created run:

$$
q_{i_{1}}=q_{i_{1}}-v_{1}, q_{i_{2}}=q_{i_{2}}-v_{2}, \ldots, q_{i_{n}}=q_{i_{n}}-v_{n} .
$$

Go to step 1.

\subsection{Synthesis of routes for each machinery set}

The problem is to optimally distribute the generated runs between the machinery sets and to determine the optimal sequence of their execution according to criteria (1) and (2).

The effect is achieved by minimizing of transportation costs by constructing predominantly cyclical routes that reduce empty runs and downtime of machinery sets [11].

This problem is determined by the expressions (1), (2) and (9) and is solved for the sequence of runs of each machinery set. The solution takes into account the connections between the schedules of the machinery sets.

To find the optimal route, a genetic algorithm is used [12-15].

The main problem of the approach presented in the article is the difficulty of determining the downtime of machinery sets at the stage of solving the LP problem.

At this stage, the model does not accurately determine the volumes of roundwood that a machinery set can transport during the planning period. As a result of this, the capabilities of some machinery sets are not fully utilized, while other machinery sets do not have time to 
complete the runs assigned to them. To solve this problem, the authors propose an approach based on the procedure for redistributing the volumes between overloaded and underloaded machinery sets in the form of post-processing.

\section{Algorithm 2 Redistribute the roundwood volumes}

Step 1. Determine the volumes of roundwood $V_{i j}$ undelivered from supply point $i, i \in I$ to demand point $j, j \in J$.

Step 2. Determine the priority $R_{i j f}$ for transporting of volume $V_{i j}$ from $i$ to $j$ for each machinery set $f, f \in F: R_{i j f}=\left(c_{i}-c_{i j f}\right)$, where $c_{j}$-assortment price per 1 cub. m. according to order $j, c_{i j f}$ - transporting costs for $1 \mathrm{cub}$. $\mathrm{m}$ of assortment from supply point $i$ to demand point according to order $j$ for machunery set $f$.

Step 3. Distribute volumes $V_{i j}$ by machinery sets in descending order of $R_{i j f}$ with the output list $V_{i j f}^{\prime}$;

Step 4. Generate "complete" and "collecting" runs according to the list $V_{i j f}^{\prime}$ using the algorithm 1. If $k \leq K_{\max }$, go to Step 1, where $k$ - number of the current iteration, $K_{\max }-$ maximum number of iterations (determined empirically).

\section{Results}

To test the developed algorithm using the real data of logging companies operating in the North-West of Russia, several dozens of transport plans were generated. For testing, the following planning indicators were selected that have direct effect on the costs:

- Transported volume (cub. m).

- Time of empty runs (hours).

- Inoperative time of machinery sets (hours).

- Utilization rate - the operating time of a machinery set divided by the planning period duration.

The planning period varied from 1 to 4 days, the number of machinery sets - from 6 to 13. The road network is similar to that described in [8]. The test results are presented in Table 1. The first value in each cell was obtained by launching the algorithm without schedule optimization (i.e. without running the genetic algorithm) and without redistributing the undelivered volume. The second value - by launching the algorithm with schedule optimization, and the third value - with schedule optimization and redistribution of the undelivered volume.

The results illustrate how the proposed algorithm allows to increase the transported volume by an average of $8 \%$. Taking into account the absolute volume $1935 \mathrm{cub}$. $\mathrm{m}$, this is a significant improvement. Also, 35\% reduction of inoperative time was achieved with an insignificant (5\%) increase of empty runs time associated with increase of the total number of runs within the planning period. The utilization rate increased by an average of $6 \%$. Thus, the algorithm proposed by the authors can significantly reduce the logistics costs of a wood harvesting company. 
Table 1. Test results.

\begin{tabular}{|c|c|c|c|c|c|}
\hline Plan & $\begin{array}{c}\text { Total } \\
\text { volume } \\
\text { (cub. m) }\end{array}$ & $\begin{array}{c}\text { Transported volume } \\
\text { (cub. m) }\end{array}$ & $\begin{array}{c}\text { Time of } \\
\text { empty runs } \\
\text { (hours) }\end{array}$ & $\begin{array}{c}\text { Inoperative } \\
\text { time (hours) }\end{array}$ & Utilization rate \\
\hline 1 & 7612 & $\begin{array}{c}7181 / 7276 / 7540 \\
(94 \% / 95 \% / 99 \%)\end{array}$ & $339 / 328 / 355$ & $122 / 116 / 110$ & $0,93 / 0,94 / 0,94$ \\
\hline 2 & 2614 & $\begin{array}{c}2476 / 2541 / 2615 \\
(95 \% / 97 \% / 100 \%)\end{array}$ & $110 / 105 / 112$ & $41 / 27 / 25$ & $0,93 / 0,95 / 0,96$ \\
\hline 3 & 3069 & $\begin{array}{c}2817 / 2901 / 2956 \\
(92 \% / 95 \% / 96 \%)\end{array}$ & $104 / 104 / 107$ & $61 / 54 / 55$ & $0,91 / 0,92 / 0,92$ \\
\hline 4 & 6520 & $\begin{array}{c}5923 / 6105 / 6249 \\
(91 \% / 94 \% / 96 \%)\end{array}$ & $264 / 277 / 274$ & $97 / 85 / 83$ & $0,93 / 0,94 / 0,94$ \\
\hline 5 & 2285 & $\begin{array}{c}1474 / 1823 / 1944 \\
(64 \% / 80 \% / 85 \%)\end{array}$ & $60 / 67 / 71$ & $57 / 17 / 6$ & $0,88 / 0,97 / 0,99$ \\
\hline 6 & 2285 & $\begin{array}{c}1379 / 1757 / 1881 \\
(60 \% / 77 \% / 82 \%)\end{array}$ & $58 / 67 / 71$ & $72 / 24 / 13$ & $0,85 / 0,95 / 0,97$ \\
\hline Total & 24385 & $\begin{array}{c}21250 / 22403 / 23185 \\
(87 \% / 92 \% / 95 \%)\end{array}$ & $935 / 948 / 990$ & $450 / 323 / 292$ & $0,90 / 0,95 / 0,96$ \\
\hline
\end{tabular}

\section{Conclusion}

An approach to the optimal timber transport scheduling is provided, including the problem statement and a multi-criteria mathematical model. The problem belongs to the class of General vehicle routing problems (GVRP) associated with the job-shop scheduling. A hybrid algorithm for solving the problem based on the decomposition method using the simplex method and the genetic algorithm is developed. Testing of the proposed approach using real data from wood harvesting enterprises showed its effectiveness.

The algorithm was implemented in "Opti-Wood" decision support system for wood harvesting planning and management, developed by Opti-Soft company [16].

\section{References}

1. A.P. Sokolov, Lesnoy Zhurnal (Russian Forestry Journal), 4, 87-103 (2015), DOI: 10.17238/issn0536-1036.2015.4.87

2. I.R. Shegelman, A.V. Kuznetsov, V.I. Skrypnik, V.N. Baklagin, Engineering journal of Don, 4 (2014)

3. J. Edmonds, Mathematical Programming, 1, 127-136 (1971), DOI: 10.1007/BF01584082

4. J.C. Beck, P. Prosser, E. Selensky, ICAPS-03, 267-276 (2003)

5. A. Goel, V. Gruhn, European Journal of Operational Research, 191, 650-660 (2008), DOI: $10.1016 /$ j.ejor.2006.12.065

6. F. Flisberg, B. Liden, M.A. Rönnqvist, Computers \& Operations Research, 36, 11221144 (2009) DOI: 10.1016/j.cor.2007.12.012

7. A.A. Lazarev, E.R. Gafarov, Job shop scheduling. Tasks and Algorithms (2011)

8. A.I. Shabaev, A.P. Sokolov, A.R. Urban, D.S. Pyatin, Resources and Technology, 1, 23-38 (2018), DOI: 10.15393/j2.art.2018.4061

9. D. Dantsig, Linear programming and its generalization and application (1966)

10. W.L. Winston, M. Venkataramanan, Introduction to Mathematical Programming: Applications and Algorithms, Volume 1. Operations Research (2002) 
11. A.P. Sokolov, Yu.Yu. Gerasimov, Functional logistics of the wood harvesting company (2014)

12. O.Z. Maimon, D. Braha, International Journal of Production Research, 36, 761-764 (1998), DOI: 10.1080/002075498193688

13. T. Vidal, T. Crainic, M. Gendreau, N, Lahrichi, W. Rei, Operations Research, 60, 611624 (2012), DOI: 10.1287/opre.1120.1048

14. D. Goldberg, R. Lingle, Proceedings of the 1st International Conference on Genetic Algorithms and Their Applications, 154-159 (1985)

15. T. Blickle, L. Thiele, A Evolutionary Computation, 4, 361-394 (1996), DOI: 10.1162/evco.1996.4.4.361

16. A.I. Shabaev, A.P. Sokolov, A.R. Urban, D.S. Pyatin, IOP Conference Series: Earth and Environmental Science, 316, 1-7 (2019), DOI: 10.1088/1755-1315/316/1/012061 